\title{
ICARUS T600: physics results and future activities
}

\author{
Andrea Zani ${ }^{1,2, a}$, On behalf of the ICARUS Collaboration \\ ${ }^{1}$ INFN, Sezione di Pavia, via Agostino Bassi 6, 27100 Pavia, Italy \\ ${ }^{2}$ CERN, route de Meyrin 385, 1217 Meyrin, Switzerland
}

\begin{abstract}
The ICARUS T600 detector has proven the effectiveness of Liquid Argon TPC technology with a successful three-year long run at the INFN Gran Sasso National Laboratories (LNGS).

ICARUS T600 LNGS data strongly contributed to the global effort in searching for neutrino oscillations mediated by sterile states. A definitive clarification of the sterile neutrino puzzle will come from the new multi-station, Short Baseline Neutrino (SBN) experiment at the Booster Neutrino Beam (BNB) at Fermilab, where a refurbished T600 will act as Far Detector.

Presently the T600 detector is located at CERN, undergoing a major overhauling which will allow its participation in the 5 years program of the Fermilab upcoming SBN project. Moreover, ICARUS T600 is also foreseen to collect data with the NUMI Beam to be later exploited in the wider picture of the DUNE Long-Baseline project, recently undertaken by the United States community. This contribution will report the present physics results obtained by the ICARUS Collaboration, as well as describe the overhauling activities and the physics program for the detector in its future deployment at Fermilab.
\end{abstract}

\section{The ICARUS T600 detector}

The ICARUS T600 detector is the largest operational Liquid Argon (LAr) Time Projection Chamber (TPC) built so far [1,2]. It was deployed and successfully operated in the Hall B of the underground Gran Sasso Laboratory (LNGS), Italy, for more than three years, exposed to both the CERN-Neutrino-to-Gran-Sasso (CNGS) beam, and cosmic rays. The CNGS facility provided an almost pure muon-neutrino beam peaked in the $10 \leq E_{v} \leq 30 \mathrm{GeV}$ range, with a $v_{e} / \bar{v}_{e}$ contamination of less than 1\%. From October 2010 to December 2012, the detector collected neutrinos corresponding to a total of $8.6 \times 10^{19} 400-\mathrm{GeV}$ Protons-On-Target (POT) with a recording efficiency exceeding $93 \%$.

The T600 detector is made of two side-by-side cryostats housing identical half-modules: each of them contains two TPCs sharing a common cathode. The half-modules internal dimensions are $3.6 \times 3.9 \times 19.6 \mathrm{~m}^{3}$; they are filled with a total of 760 tons of ultrapure Liquid Argon. Each TPC has maximal drift length of $1.5 \mathrm{~m}$, over which a constant electric field $E_{D}=500 \mathrm{~V} / \mathrm{cm}$ is present. Charged particles interacting in the TPC active volume produce both scintillation light and ionization electrons: the latter are drifted by the field to the

a e-mail: andrea.zani@cern.ch 
anode, which is made by three parallel wire planes facing the drift volume, $3 \mathrm{~mm}$ apart and with a $3 \mathrm{~mm}$ pitch. Each wire plane is oriented at a different angle $\left(0^{\circ},+60^{\circ},-60^{\circ}\right)$ with respect to the horizontal direction, for a total of about 54000 instrumented wires. Seventyfour 8" photomultipliers (PMTs) in total, positioned behind the wires, collect the produced scintillations light, thus yielding information about the track arrival time. In order to collect the UV light generated in liquid Argon (at $\lambda=128 \mathrm{~nm}$ ), the PMT windows are coated with Tetra-phenyl Butadiene, TPB, a wavelength shifter re-emitting photons at around $\lambda=430$ nm.

The measured drift time, combined with the electron drift velocity $\left(v_{D}=1.59 \mathrm{~mm} / \mu \mathrm{s}\right.$ at $\left.E=E_{D}\right)$, provides the event coordinate in the drift direction; the composition of the three views from the TPC wires instead yields the track position in the anode plane. Matching these pieces of information allows obtaining a full 3D reconstruction of the tracks, with a precision of few $\mathrm{mm}^{3}$.

The electronic chain is designed to allow for continuous read-out, digitization (10-bit FADC) and independent waveform recording for each wire signal of the four TPCs; 400 ns non-synchronous sampling is applied on the waveforms. The trigger relies on detection of the UV scintillation light produced by the primary track, in coincidence with the CERN-SPS proton extraction time for the CNGS beam.

Two main items may be highlighted, which assured ICARUS T600 excellent technical and physical results: the capability to achieve unprecedented levels of liquid argon purity, and LAr unique features, which allow for highly-efficient $e / \gamma$ separation. Continuous gas $\left(2.5 \mathrm{~m}^{3} / \mathrm{h}\right)$ and liquid $\left(100 \mathrm{~m}^{3} / \mathrm{d}\right)$ recirculation, as well as standard commercial Hydrosorb/Oxysorb ${ }^{\mathrm{TM}}$ filters, were used to reduce the concentration of electronegative impurities $\left(\mathrm{O}_{2}, \mathrm{~N}_{2}, \mathrm{H}_{2} \mathrm{O}\right)$ : this has to be kept far below the value of $0.1 \mathrm{ppb}-\mathrm{O}_{2}$ equivalent, to ensure ionization charges travel through the entire drift volume. Such system allowed obtaining, during the LNGS run, an average electron life-time $>7 \mathrm{~ms}$ (max charge attenuation along the drift: 12\%), and a maximum value of $16 \mathrm{~ms}$ (with a new liquid pump), which translates into an impurity concentration lower than 20 ppt $\mathrm{O}_{2}$ equivalent, as shown in figure 1 [3]. Such a result represents a fundamental step towards the construction of a new generation of detectors, with drift paths many meters long.

The precise spatial and calorimetric reconstruction achievable in the T600, along with the possibility to measure local energy depositions $(d E / d x)$ wire by wire, allows the recognition of single- and double-ionising particles events: it is thus possible to efficiently perform $e / \gamma$ separation, and full reconstruction of $\pi^{0}$ 's. Such feature ensures therefore negligible contamination of $\pi^{0}$ s from $N C$ (Neutral Current) and $v_{\mu} C C$ in selected $v_{e}$-event samples, which is fundamental for the searches of non-standard $v_{\mu} \rightarrow v_{e}$ oscillations.

\section{Sterile neutrino searches}

World experimental observations of neutrino oscillations have so far established a consistent paradigm consisting of three neutrino flavors $\left(v_{e}, v_{\mu}, v_{\tau}\right)$ resulting from the mixing of three mass eigenstates $\left(v_{1}, v_{2}, v_{3}\right)$ with relatively small mass differences: $\Delta m_{31}^{2} \simeq 2.4 \times 10^{-3} \mathrm{eV}^{2}$ and $\Delta m_{21}^{2} \simeq 7.5 \times 10^{-5} \mathrm{eV}^{2}[4]$. Nonetheless, more recent experiments have reported several anomalies which, if confirmed, could hint at the presence of additional non weakly interacting, hence sterile, neutrino flavours with larger mass-squared differences participating in the mixing. On the other side, anyway, Big Bang cosmology and the data from the Planck satellite [5] still favour models with at most one further $v$-flavour, its mass being $<0.4 \mathrm{eV}$. 


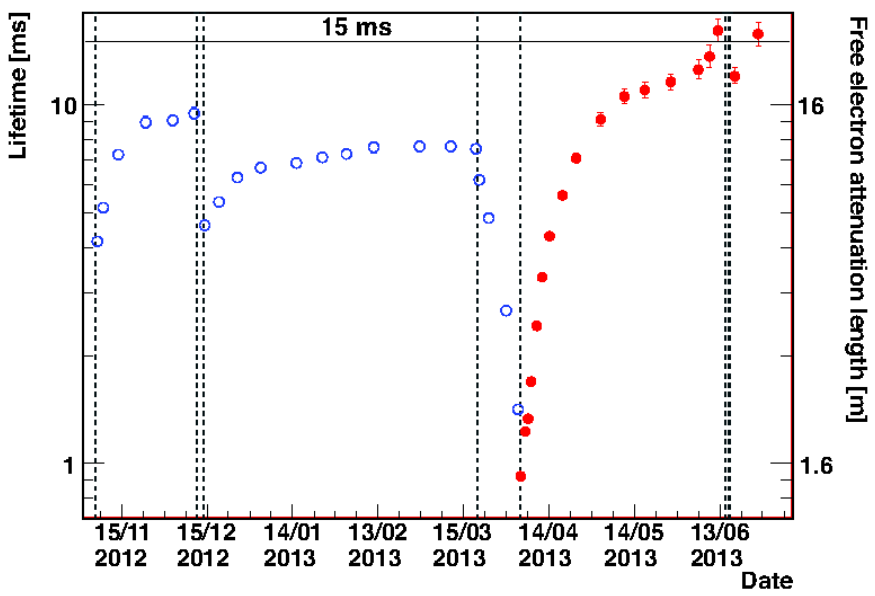

Figure 1. Electron life-time $\tau_{e}$ in the T600 East half-module, as a function of time [3]. Stops of recirculation due to pump maintenance are evident from the decrease in purity. The installation of a new faster pump from April 2013 translates in a much larger purification speed (cfr. slope of data points).

Two distinct classes of neutrino anomalies have been reported so far: first, the apparent disappearance signal in low energy $\bar{v}_{e}$ from nuclear reactors, beyond the expected $\theta_{13}$ effect [6], and from Mega-Curie radioactive $v_{e}$-sources in the Gallium experiments $[7,8]$ originally designed to detect solar neutrinos. On the other hand, there is evidence for an $v_{e} / \bar{v}_{e}$ excess in experiments using muon beams from particle accelerators [9, 10] (the LSND and MiniBooNE anomalies).

All these data may be interpreted by postulating the existence of at least one additional sterile neutrino state with mass $\lesssim 1 \mathrm{eV}^{2}$. However, an issue with such explanation is due to some tension between appearance and disappearance data, in particular since no evidence of $v_{\mu}$ disappearance is observed in the $\Delta m_{41}^{2} \sim 1 \mathrm{eV}^{2}$ region.

\subsection{Search for LNSD-like effect with ICARUS and the CNGS beam}

The ICARUS T600 detector has conducted a search for a LSND-like, $v_{e}$-appearance effect during its run at LNGS, with the CNGS beam, characterised by $L / E_{v} \sim 36.5 \mathrm{~m} / \mathrm{MeV}(L \simeq 732$ $\mathrm{km})$. This is quite far from the value $\sim 1$ of LSND, nonetheless a search was performed, on a sample of $2650 v$-events from the CNGS beam $\left(7.93 \times 10^{19}\right.$ POT $)$. The expected background takes into account intrinsic $v_{e}$ contamination, $\theta_{13}$-driven oscillations and decays of $\tau$ 's from $v_{\mu} \rightarrow v_{\tau}$ oscillations: weighting for the identification efficiency, $8.4 \pm 1.1 v_{e}$ events can be predicted in the sample. The actual number of observed events $[11,12]$ is seven, which yields no exotic oscillation evidence (see f.i. fig 2).The following limits on exotic oscillations probability can be therefore derived $[11,12]$, as:

$$
P\left(v_{\mu} \rightarrow v_{e}\right) \leq 3.85 \times 10^{-3}(90 \% \text { C.L. }) \quad P\left(v_{\mu} \rightarrow v_{e}\right) \leq 7.60 \times 10^{-3}(99 \% \text { C.L. })
$$


The corresponding exclusion plot is reported in figure 3. Such result is corroborated by the OPERA experiment [13]. Taking into account these limits and all other world results, there still remains a region in the parameter space, centered around $\Delta m_{41}^{2} \sim 0.5 \mathrm{eV}^{2}$ and $\sin ^{2} 2 \theta_{14} \sim 0.005$, where overall agreement holds between the described negative results and the positive signals from LSND and MiniBooNE.

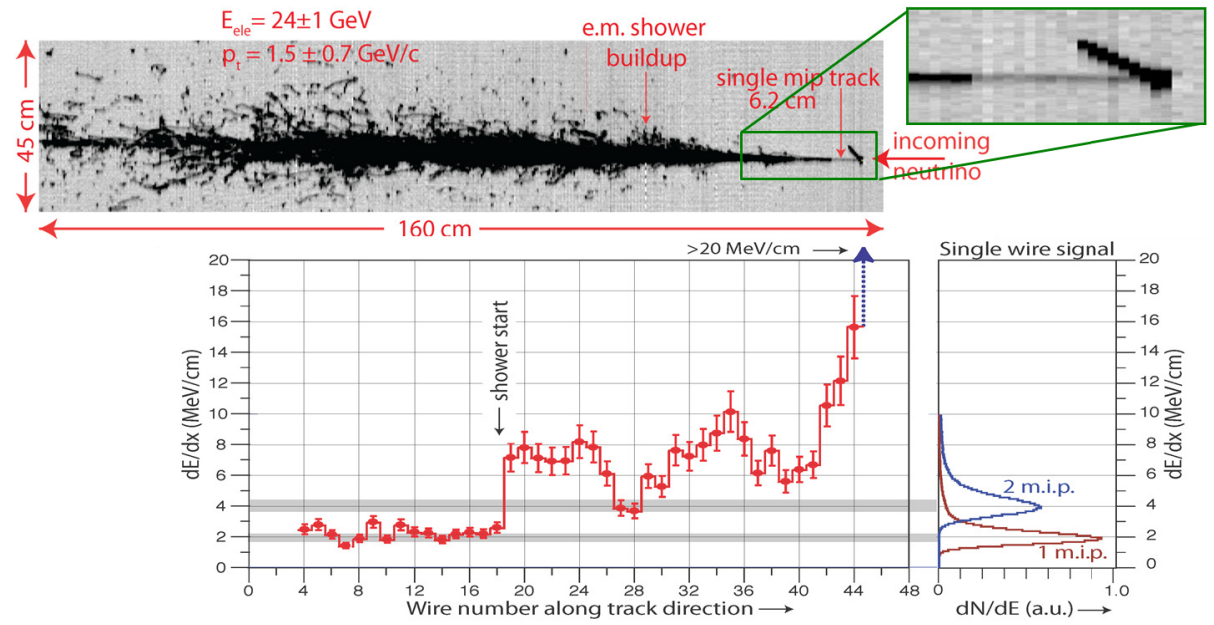

Figure 2. Example of $v_{e} C C$ interaction from the CNGS beam The single-ionizing particle behaviour is clearly visible before the development of the shower, both in the TPC Collection view and in the $d E / d x$ analysis below.

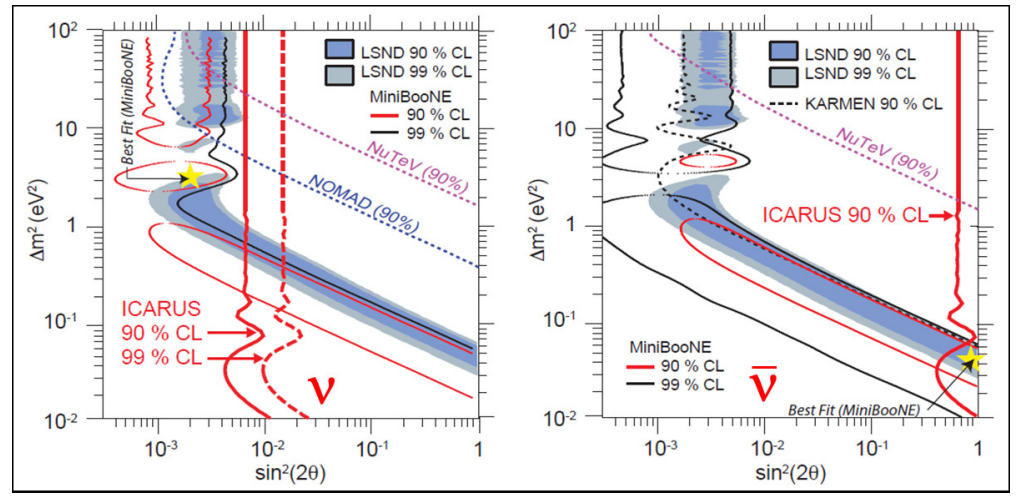

Figure 3. ICARUS limits on the search for non-standard $v_{\mu} \rightarrow v_{e}$ oscillations with CNGS beam, compared with previous results. OPERA analysis aligns with ICARUS findings. 


\subsection{The Short Baseline Neutrino (SBN) program at FNAL}

In order to definitely solve the sterile neutrino puzzle, a whole new short baseline program is being developed, involving diffrerent Collaborations and a global effort. The Short Baseline Neutrino program [15], based at the Fermilab National Accelerator Laboratory (FNAL), foresees the deployment on the Booster Neutrino Beam (BNB) of multiple LAr-TPCs, namely SBND (82 $\mathrm{t}$ active volume), MicroBooNE (89 t) and ICARUS T600 (476 t), at a distance of 150, 470 and $600 \mathrm{~m}$ respectively. Such configuration will allow simultaneous observations of $v$-interactions at different distances, thus yielding separate identification of the $\Delta \mathrm{m}_{14}^{2}$ and $\sin ^{2}\left(2 \theta_{14}\right)$ parameters. The detectors will operate at $L / E_{v} \sim 1$ and collect millions of events, thus being able to identify effects at the percent level. The expectation is to have perfectly superimposed neutrino spectra at the different stations, in case of oscillation absence: on the contrary any difference may imply a new physics signal. Furthermore the possibility to intervene on beam optics, flipping between neutrino and anti-neutrino modes, assures the chance to disentangle appearance/disappearance effects.

A significant effort has been produced jointly by the three Collaboration to evaluate with MonteCarlo simulations the sensitivity of the program to appearance/disappearance signals, also considering one new channel (at least for this type of detector), i.e. $v_{\mu}$ disappearance, connected to $v_{e}$ appearance via the relation:

$$
\sin ^{2}\left(2 \theta_{\mu e}\right) \leq \frac{1}{4} \sin ^{2}\left(2 \theta_{\mu x}\right) \sin ^{2}\left(2 \theta_{e x}\right)
$$

The sensitivity evaluation has been made by considering a joint exposure of three years $\left(6.6 \times 10^{20}\right.$ POT from the BNB), or six years for MicroBooNE, and this should allow covering the LSND 99\% CL region at the $5 \sigma$ level, at the least for the $v_{e}$ channel (see fig. 4 ). On the other hand, the sensitivity in the $v_{\mu}$ disappearance channel at $600 \mathrm{~m}$ distance would be limited to the very-low energy bins $(0.2-0.4 \mathrm{GeV})$ for small mass differences $\left(\Delta \mathrm{m}_{14}^{2}<0.5 \mathrm{eV}^{2}\right)$ : for this reason, it is being considered to move, at a later stage, one of the two T600 half-modules further away from the beam target, at $1500 \mathrm{~m}$ distance, to enhance the effect. However such implementation is still at the level of a consideration and a decision is still to be made.

\subsubsection{Cosmic background on the surface and mitigations}

The US program will have to face a rather new challenge for LAr-TPCs: the operation at shallow depths. All detectors will be deployed at few meters below ground level, covered by a three-meter concrete shielding. Nonetheless still several cosmic rays will cross the LAr active volumes during data taking: in particular for the T600 12 muon tracks per halfmodule are expected in the $1 \mathrm{~ms}$ drift readout window, as estimated during the 2001 test-run performed at sea-level in Pavia [1]. This situation is completely different with respect to Gran Sasso, where each trigger contained only one event, either from beam or cosmic background: therefore new solutions must be devised to tag cosmic events, and to reconstruct position in space and timing of tracks, in order to associate them (or not) to the beam trigger.

The most serious background source is due to photons associated to cosmic muons: they can mimic $v_{e} C C$ interactions by producing electrons via Compton effect or pair production, and are most dangerous especially when they enter the active volume while their associated $\mu$ does not (as it may cross the non-active LAr region)

Some solutions are already planned for background mitigation and are being implemented: 


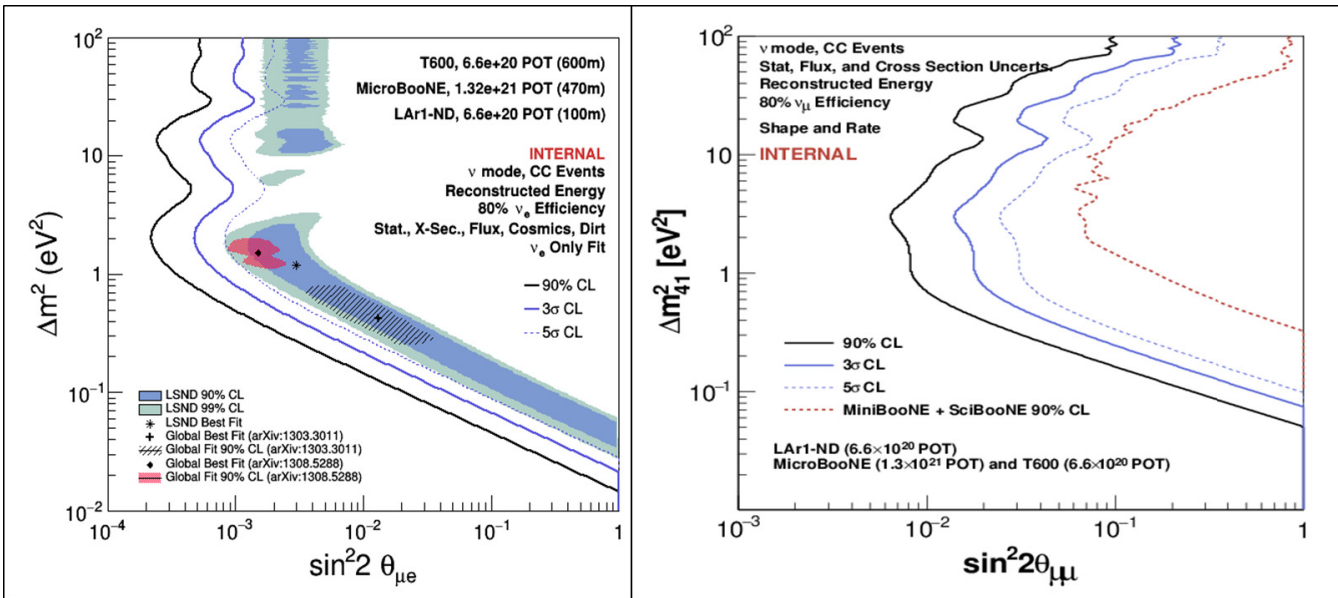

Figure 4. Expected sensitivity for the SBN program in the $v_{e}$-appearance (left) and $v_{\mu}$-disappearance channels (right), for three years of data taking and $6.6 \times 10^{20}$ POT from the Booster Neutrino Beam.

- software-wise, a first cut on $\gamma$ 's associated to muons can be made with geometrical selections directly on the TPC images: f.i., by defining a minimum distance between the $\gamma$ and the $\mu$ (a cylinder around the muon track) which corresponds to associated particles.

- The achievement of $1 \mathrm{~ns}$ timing accuracy for light collection with the internal PMT system will allow exploiting the bunched structure of the BNB, which is characterized by 2 ns spills every 19 ns. For this purpose, the light collection system is being significantly upgraded, as it will be further detailed in section 3 .

- The implementation of a so-called Cosmic Ray Tagger (CRT), i.e. an active layer surrounding as much as possible the TPC and yielding time information of external tracks. The combination of timing from both the CRT and internal PMT system will allow recognising cosmic tracks, and, with the mentioned timing accuracy, distinguish them (that enter the LAr volume) from long muons in $v_{\mu} C C$ beam events (going outward, possible source of auto-veto). Presently the CRT design foresees two-layer scintillator panels, for x-y position reconstruction, with embedded optical fibers and read by Silicon PhotoMultipliers (SiPMs). The panels will be installed and integrated in the outer supporting structure (warm vessel) of the T600.

It is important to note that the CRT, as well as all the software tools to be implemented for neutrino events reconstruction and selection, are being actually developed jointly by the three Collaborations, as they are common to the whole SBN program.

\section{Overhauling activities at CERN}

Before heading to FNAL, the T600 detector has to undergo a significant overhauling procedure [15]. This will allow introducing technological developments, such as new state-of-the-art, better performing components, while at the same time maintaining the already achieved performance. This activity is taking place at CERN, and it is regulated by a Memorandum 
of Understanding between CERN and INFN. Completion of the refurbishment is scheduled for the end of 2016, so that detector installation at FNAL can start in 2017.

The overhauling activity at CERN covers important systems of the T600, in particular:

- flattening of the existing cathode panels, to get improved planarity;

- new cold vessels made of Aluminum extruded profiles, and new purely passive insulation;

- refurbished cryogenic plant, organised in skids for independent testing/shipping;

- upgraded light collection system;

- faster warm electronic and read-out chain.

The last two items, objects of significant R\&D, are further detailed in the following.

As already mentioned in section 2.2.1, light collection in the new SBN program will be fundamental, both for trigger and for tagging of cosmic background tracks. Therefore, a significant upgrade is planned for the PMT system: new 8" Hamamatsu photomultipliers (PMTs) will be installed, 90 per TPC, yielding larger Quantum Efficiency (QE), around 7\%, and larger photocathodic coverage, of the order of $5 \%$, with respect to the past [1]. The chosen 10-dynode, R5912 Hamamatsu PMTs are characterised by high linearity and nanosencondhigh time resolution, and will be equipped with new voltage dividers and shielding grid, to avoid induced spurious signals on the TPC wires, as seen during the LNGS run.

The high time resolution of these tubes allows exploiting the bunch structure of the BNB beam, effectively selecting events from the spill; on the other hand the improved coverage and QE leads to high spatial resolution, which in turn allows localization and selection of events in the active volume. A full simulation of the new light collection system has been developed, to optimize the lay-out and number of PMTs: 90 samples per TPC provide longitudinal resolution $<0.5 \mathrm{~m}$; furthermore, by setting a 10 phe threshold on the detected fast light component, it is possible to reduce the residual error on event localization to better than $\pm 20 \mathrm{~cm}$, with wrongly reconstructed events limited to the detector boundaries. The same simulation was later developed for the chosen configuration, with the implementation of a neural network used to identify events and separate straight tracks (mainly cosmic muons) from electromagnetic showers, possibly due to $v_{e} C C$ beam events. The network was tested with $\mathrm{MC}$ events and it was possible to obtain very high efficiency, with mis-identification at the level of few percent.

The ICARUS electronic chain is being remade from scratch, with the idea to maintain the same structure, but with newer, higher-performance components: the architecture is based on analogue low noise warm front-end amplifiers, multiplexed 10-bit 2.5 $\mathrm{MHz}$ ADCs (resulting in 400 ns non-synchronous sampling) and digital VME modules for local storage, data compression and trigger information. With this configuration it was achieved, during the LNGS run, a signal-to-noise ratio better than 10 and a $\sim 0.7 \mathrm{~mm}$ single-point resolution. While this structure is still very well suited for operation with even larger TPCs, it is important to insert newer components, and improve some aspects:

- introduction of serial ADCs, one per channel, to allow for synchronous sampling;

- adoption of modern serial bus architecture, with optical links for Gbit/s transmission rate;

- new compact design, with the digital part residing in a single FPGA, and the resulting boards housed in newly-designed mini-crates directly mounted on the flanges on top of the detector (significant decrease of volume). 
New electronic boards containing all the mentioned improvements are being developed by the Padova group in close collaboration with CAEN industry. At the same time, the ICARUS group at CERN has tested for a few months a different solution, i.e. cold front-end boards developed by BNL and already in use by MicroBooNE. The results were quite satisfying, with the achievement of a signal-to-noise ratio $~ 20$ on both Induction and Collection wire planes, but the solution was eventually dropped, due to short time available for installation.

\section{Conclusions}

The ICARUS T600 detector has operated smoothly for three years in the Gran Sasso underground laboratories, establishing the success of Liquid Argon TPC technology for large volumes, and paving the way for multikton-scale detectors. During the LNGS run a search was performed for non-standard $v_{\mu} \rightarrow v_{e}$ oscillations driven by supposed sterile neutrino states, but no evidence of oscillation was actually observed.

The ICARUS Collaboration is now embarking in the SBN program at FNAL, specifically dedicated to sterile neutrino searches. The program brings together three experiments (SBND, MicroBooNE and ICARUS), on the Booster Neutrino Beam, to cover in 5 years the $\left(\Delta m_{14}^{2}, \sin ^{2} 2 \theta_{14}\right)$ parameter space and finally solve the puzzle. At the same time the ICARUS detector, also positioned off-axis on the NuMi beam, will collect $v_{e} C C$ events, to provide initial data for the future Long Baseline FNAL experiment, LBNF/DUNE (former LBNE) [17].

Before heading to the US, the T600 was moved to CERN, where it is undergoing a major technological overhaul, that will prepare the detector for its deployment at FNAL.

\section{References}

[1] Amerio S. et al. (ICARUS Collab.), Nucl. Instr. and Meth. A 527, 329-410 (2004)

[2] Rubbia C. et al. (ICARUS Collab.), JINST 6, P07011 (2011)

[3] Antonello M. et al. (ICARUS Collab.), JINST 9, P12006 (2014)

[4] Olive K.A. et al. (Particle Data Group), Chin. Phys. C, 38, 090001 (2014)

[5] Planck Collaboration, A\&A, 571, A16 (2014)

[6] Mention G. et al., Phys. Rev. D 83, 073006 (2011) and reference therein

[7] Abdurashitov J.N. et al., Phys. Rev. C 80, 015807 (2009)

[8] Kaether F., Hampel W., Heusser G., Kiko J. \& Kirsten T., Phys. Lett. B 685, 47 (2010)

[9] Aguilar A. et al., Phys. Rev. D 64, 112007 (2001)

[10] Aguilar-Arevalo A. A. et al. (MiniBooNE Collab.), Phys. Rev. Lett. 110, 161801 (2013)

[11] Antonello M. et al. (ICARUS Collab.), Eur. Phys. J. C73, 2345 (2013)

[12] Antonello M. et al. (ICARUS Collab.), Eur. Phys. J. C73, 2599 (2013)

[13] Agafonova N. et al. (OPERA Collab.), JHEP 1307, 004 (2013)

[14] Armbruster B. et al. (KARMEN Collaboration), Phys. Rev. D 65, 112001 (2002)

[15] SBND, MicroBooNE and ICARUS Collaborations, SBN-doc-269-v5, arXiv:1503.01520v1 [physics.ins-det] (2015)

[16] http://www.gtt.fr

[17] http://www.dunescience.org/ ; Adams C. et al. (LBNE Collaboration), FERMILABPUB-14-022, arXiv:1307.7335v3 [hep-ex] (2013) 